\title{
Performance Evaluation of Bluetooth Low Energy Technology under Interference
}

\author{
Heikki Karvonen, Konstantin Mikhaylov, \\ Dinesh Acharya and Md. Moklesur Rahman \\ Centre for Wireless Communications, University of Oulu, Finland, \\ heikki.karvonen@oulu.fi
}

\begin{abstract}
This paper focuses on analytical and experimental performance evaluation of the Bluetooth Low Energy (BLE) technology. Studies have been conducted in indoors case relevant to healthcare and medical scenarios. Performance of the recently developed BLE 5 coded technique is compared to BLE 4 which is currently the most used technology in commercial wireless healthcare and medical devices. This new improved BLE version may continue fostering the success of BLE use in those application scenarios as well as enable novel Internet of Things (IoT) solutions. The main goal of this work was to evaluate the packet error rate (PER) performance of BLE under ZigBee interference, since it is envisaged, that coexistence problems may arise with the further growth of number of the different IoT devices deployed. In the paper we first develop an analytical model to characterize the PER of BLE link with varying distance to interfering nodes. Then we conduct a series of practical measurements using the Nordic Semiconductor nRF52840 chipset, which supports the new BLE 5 coded features. Our results show that ZigBee interference is very harmful for BLE communication when operating at the same frequency band, i.e., assuming worst-case scenario. The proposed model can be used to evaluate PER of BLE in various interference scenarios to get insight of communication reliability which is very important specifically for healthcare and medical applications.
\end{abstract}

Key words: wireless coexistence, BLE 5, ZigBee, packet error rate (PER), Internet of Things, healthcare and medical applications.

\section{Introduction}

The use of wireless sensor devices has been continuously increasing during recent years thanks to rising success of Internet of Things (IoT) applications. Sensor devices can be used in various scenarios, e.g., smart factories and homes, environmental monitoring, autonomous traffic, medical and healthcare applications. Wireless body area networks 
(WBANs) are used in the context of smart healthcare applications, operating in hospitals or homes, as well as for versatile sport and fitness activities. WBAN sensors can be also connected to Internet, being a one specific IoT use case which is gaining an increasing business interest [1], [2].

IoT applications require low-power wireless communication solutions since most of the use cases imply long lifetime for the sensor nodes without battery replacement, or even using only the energy scavenged from the operation environment. There are various low-power communication technologies that have been proposed for wireless sensor nodes. The most well-known ones are Bluetooth Low Energy (BLE) [3] and IEEE Std. 802.15.4 [4] (ZigBee [5]). Specifically for WBAN purposes has been defined IEEE Std. 802.15.6 [6] and ETSI SmartBAN [7]. In [8] it was found that BLE stands out as the most widely used in current commercially available products in healthcare and medical applications. Above mentioned technologies operate in the industrial, scientific and medical (ISM) $2.4 \mathrm{GHz}$ band, which is available worldwide enabling interoperability in different regions. IEEE Std. 802.15.6 defines solutions also to sub-GigaHertz bands as well as for ultra wideband (UWB) up to $10.6 \mathrm{GHz}$. Today, the $2.4 \mathrm{GHz}$ band is rapidly becoming congested due to the presence of several other wireless technologies such as IEEE Std. 802.11 (Wi-Fi), and most recently, the upcoming unlicensed LongTerm Evolution (LTE) solutions (LTE-U) [9]. Therefore, the coexistence issues may arise as the number of devices operating at that band increases rapidly.

It is important to evaluate the wireless communication performance in the congested scenario at $2.4 \mathrm{GHz}$ ISM band especially for applications which require reliable communication as is the case in many healthcare and medical scenarios. In [10] authors studied analytically the packet error rate (PER) of BLE under interference of ZigBee, Wi-Fi and BLE 5 in hospital scenario. Here will be used experimental measurements to verify the analytical model findings in case of BLE under ZigBee interference. We conduct our measurements and report the results not for BLE 4 only, but also for recently published BLE 5 coded $(\mathrm{S}=8)$ mode.

The structure of the rest of the paper is as follows. Section 2 briefly describes the specifics of the BLE technology. Analytical model for PER calculation is introduced ion Section 3. Measurement devices and set-up are described in Section 4. Section 5 introduces the analytical and experimental results. Conclusions are given in Section 6.

\section{Features of the BLE technology}

The low-power version of Bluetooth, BLE 4, has been in use since June 2010 and today it can be found in almost every smartphone, tablet, and laptop in the market in addition to a large set of other wireless devices. The most recent version, Bluetooth 5, was introduced in December 2016 [11] with the first commercial development kits becoming available in early 2017 . The long-range and mesh features has made BLE 5 very suitable for versatile IoT applications. The official announcement of BLE 5 states that the increase in range is up to 4 times compared with BLE 4.2 [12]. In the rest of this section we will focus on the most important changes of BLE 5 compared to BLE 4.2. 
The problem of improving the communications range and the maximum throughput has been addressed in BLE 5 specification by introducing three new physical layer (PHY) options. In addition to the $1 \mathrm{Mbit} / \mathrm{s}$ Gaussian frequency shift keying (GFSK) of BLE 4 (named in Bluetooth v 5.0 core specification LE 1M), the BLE 5 specifies a 2 Mbit/s GFSK PHY (named LE 2M) for short range high-speed transmission and two coded PHY (referred to as LE Coded) with payload coded at $500 \mathrm{kbit} / \mathrm{s}(\mathrm{S}=2)$ or 125 $\mathrm{kbit} / \mathrm{s}(\mathrm{S}=8)$. The LE coded PHYs are modulated using GFSK at $1 \mathrm{Msym} / \mathrm{s}$ rate, but the payload data are coded in two stages: first by forward error correction convolutional encoder and then spread by the pattern mapper. In theory, this enables to improve the link budget of a coded transmission by over $5 \mathrm{~dB}$ and $12 \mathrm{~dB}$ compared to LE $1 \mathrm{M}$ for LE coded at $500 \mathrm{kbit} / \mathrm{s}$ and $125 \mathrm{kbit} / \mathrm{s}$, respectively. Note, that only the support of LE $1 \mathrm{M}$ PHY is mandatory.

Another change introduced to improve the communication range is the increase of the maximum transmit power of a BLE from $10 \mathrm{dBm}(10 \mathrm{~mW})$ to $20 \mathrm{dBm}(100 \mathrm{~mW})$. Unfortunately, due to the transmit power restrictions imposed by the frequency regulations, this higher transmit power does not provide any benefit for some regions (namely, EU, Japan and Korea). The maximum link layer protocol data unit (PDU), increased in BLE 4.2 from 39 to 257 octets, stayed at this level also in BLE 5. The problem of coexistence of devices in the $2.4 \mathrm{GHz}$ band has been addressed in BLE 5 by introducing the special interface proving signaling and messaging mechanisms between collocated Bluetooth and other mobile wireless standard radios.

In addition to these changes, the functionality of the broadcasting channels in BLE 5 has been substantially enriched by the introduced extended advertising feature. First, the concept of the secondary advertising channels which are co-allocated with the BLE data channels was introduced. The format of the advertising packets used in the secondary channels has been reworked enabling them to carry up to 255 octets of PDU (compared with 37 octets allowed in the primary channels of BLE 4) and even to support fragmentation. Another interesting feature enabled in the BLE 5 is the periodic advertisements. Hopping between the secondary channels in a predefined pseudo-random sequence, a periodic advertiser broadcasts the packets, with PDU of up to 255 octets, at regular intervals of time ranging from $7.5 \mathrm{~ms}$ to almost $82 \mathrm{~s}$. Importantly, a scanner device may synchronize with one or even several non-overlapping (in time) periodic advertisers and get the data from all of them. This equips BLE 5 with a more efficient and reliable solution for data broadcast than the one possible with BLE 4. Note, that the support of periodic advertisements and extended advertising features is optional.

Importantly, the BLE 5 is backward-compatible with the earlier versions of BLE all the discussed features are optional and are not necessarily needed to be supported. Nonetheless, as one can easily see, they can substantially increase the communications range or throughput or enable new modes of operation. Due to this fact, in the marketing materials of Bluetooth SIG [12], the BLE 5 is claimed to provide double bandwidth, up to four times higher range and up to 8 times broadcasting capacity compared to BLE 4.2. However, it must be noted that the improved data rate and communication range cannot be achieved at the same time since they are provided by different PHY modes. 


\section{$3 \quad$ Analytical model}

Here will be introduced an analytical model that can be used to compute the PER of BLE uncoded mode under interference. The developed model takes into account interference of multiple nodes by aggregating the signal power coming from them.

There are several path loss models $(2.4 \mathrm{GHz})$ proposed for indoor environments. For line-of-sight (LOS) scenarios the path loss equation is typically defined, as a function of distance $d$, as

$$
P L(d)=P L_{0}+10 n \log _{10}\left(\frac{d}{d_{h 0}}\right),
$$

where $n$ is the path loss exponent and $d_{h o}$ is the reference distance at which the reference path loss $P L_{0}$ is measured. In [13], measurements were conducted and a path loss model was specifically developed for a hospital indoor environment. Authors found in [13] that for a LOS hospital room case $n=1.2$, which we are using in our calculations in the rest of the paper.

The signal to interference ratio (SIR) at the affected receiver under multiple radios interference can be computed as [14]

$$
\operatorname{SIR}[d B]=\left(P_{S}-P L(L)-\sum_{i=1}^{N}\left(W_{D, I} P_{i}-P L\left(d_{i}\right)\right),\right.
$$

where the desired signal's power is $P_{S}$ and $P_{i}$ is the power of the $i$ :th interferer (in $\mathrm{dB}$ ). The distance to the desired signal's transmitter is $L$, and $d_{i}$ is the distance to the $i:$ th interferer. $W_{D, I}$ is a coefficient that limits the interfering power to the bandwidth occupied by the technology being interfered with. It is defined in [15] as follows

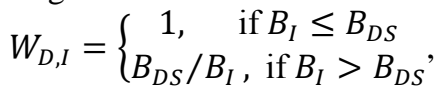

where $B_{I}$ is the bandwidth of the interferer signal and $B_{D S}$ is the bandwidth of the target node receiver filter. For this study the BLE is assumed to use GFSK modulation with bandwidth $1 \mathrm{MHz}$, bit rate $R_{b}=1 \mathrm{Mbit} / \mathrm{s}, B T=0.5$ and modulation index $h=0.5$. For non-coherent demodulation, the symbol error rate (SER) is calculated as [14] [15]

$$
S E R=\frac{1}{2} e^{-E_{S} / 2 N_{0}}=\frac{1}{2} e^{-S I R / 2},
$$

where $E_{\mathrm{s}}$ is the energy per symbol, $N_{0}$ is the noise power spectral density per Hz. In Eq. $4, E_{\mathrm{S}} / N_{0}=E_{\mathrm{b}} / N_{0}=B_{\mathrm{DS}} / R_{\mathrm{b}} * \mathrm{SNR}$, where $E_{\mathrm{b}}$ is the energy per bit and SNR is the signal-to-noise ratio. When replacing the noise power with the interference power after the receiver filter, SNR is equivalent to SIR [15], which has been inserted to Eq. (4).

Here we assume a worst-case scenario where full collision of interfering packets and the useful packet occurs, therefore SER can be assumed to be same for each transmitted symbol of the BLE packet. The PER for the affected BLE link can be calculated as

$$
\text { PER }=1-(1-\varepsilon)^{K},
$$

where $K$ is the length of the packet of the desired signal and $\varepsilon$ is the SER that can be calculated using Eq. 4. 


\section{$4 \quad$ Measurement devices}

In our measurements, one of the first commercial chipsets that support BLE 5.0, the nRF52840 [16] from Nordic Semiconductor, was used. The nRF52840 is a system on chip (SoC) integrating a multiprotocol $2.4 \mathrm{GHz}$ transceiver with an ARM Cortex-M4F based microcontroller. The chipset was programmed with S140 SoftDevice v6.0.0, which is a precompiled and linked binary software implementing BLE protocol developed by Nordic Semiconductor.

In the experiments we have used two nRF52840 Preview DK development kits shown in Figure 1. The firmware for them was developed in this work based on the ATT_MTU Throughput Example of the nRF5 software development kit (SDK) v15.0.0. One of the boards was programmed to act as an advertiser, and the other one - as scanner. The BLE physical layer to be used by the boards (i.e., LE 1M, LE 2M or LE Coded) can be selected during the startup using the control buttons.

The methodology of our experiments was as follows. After placing the BLE boards in the specified locations, the scanner board was connected to a computer via serial over USB interface, configured to operate using the required PHY layer option, and forced to continuously scan a single advertisement channel. Approximately every second the scanner reported via serial interface the number of the received advertisements from the advertiser board, as well as the received signal strength indicator (RSSI) and the sequence identifier of the last advertisement it has received. Once the scanner board was activated, the advertiser was powered up and its PHY layer was configured. The advertiser started periodically sending the advertisements, each of which contained a unique sequence number. At the end of the experiment the PER was calculated from the total number of the packets received by the scanner and the sequence number of the last received packet.

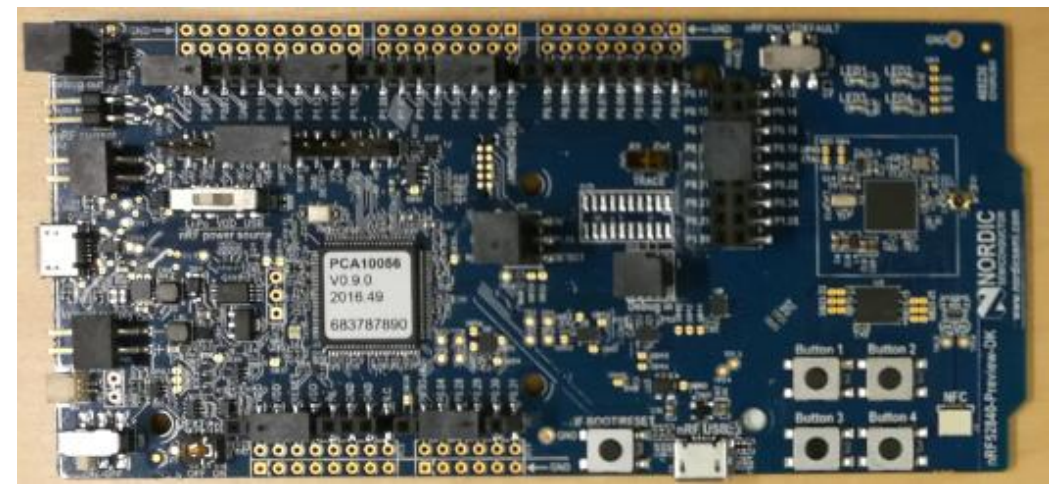

Figure 1. nRF52840 Preview DK device used for measurements.

In order to introduce the ZigBee interference to BLE communication, we used in our measurements the CWC-MOD-POW platforms (version two) [17] [18] illustrated in Figure 2. These boards are built around Texas Instruments' CC2650 multi-standard 
system-on-chip [19].The core middleware is based on CWC CC2650 IEEE Std. 802.15.4 proprietary driver and firmware, developed in TI CCS 7.4.0.00015 IDE. ZigBee nodes are equipped with an external antenna, Taoglas FXP70 [20]. The nodes were configured to start spamming the ZigBee packets with maximum possible payload without using any form of listen before talk at the same channel where BLE devices operate immediately after power up. The time between two sequential packets (due to radio re-configuration and uploading of the new packet) was well below $1 \mathrm{~ms}$. In order to ensure continuity of the interferences multiple ZigBee interferers were used in our experiments.

In our measurement the BLE boards were set at the same height $(1 \mathrm{~m})$ so that antennas were pointing each other creating a LOS link. Three interfering ZigBee nodes were set around the BLE receiver, all at the same distance (Case $1=4 \mathrm{~m}$ and Case $2=6 \mathrm{~m}$ ) to BLE receiver antenna. Different BLE link lengths were used (4 - $11 \mathrm{~m})$ and number of transmitted and received packets was recorded for 10 minutes period (resulting in at least 10000 BLE packets being sent).

Measurement environment was a restaurant at the University of Oulu during a time when there were not customers. This environment appeared to provide similar path loss as the hospital room LOS model introduced in [15] with path loss exponent $n=1.2$. Therefore, this was a good environment to obtain results that can be applied also to hospital case. Spectrum sniffers were used to find out that there was not interference from WiFi or Bluetooth at the same band were our measurements were conducted.

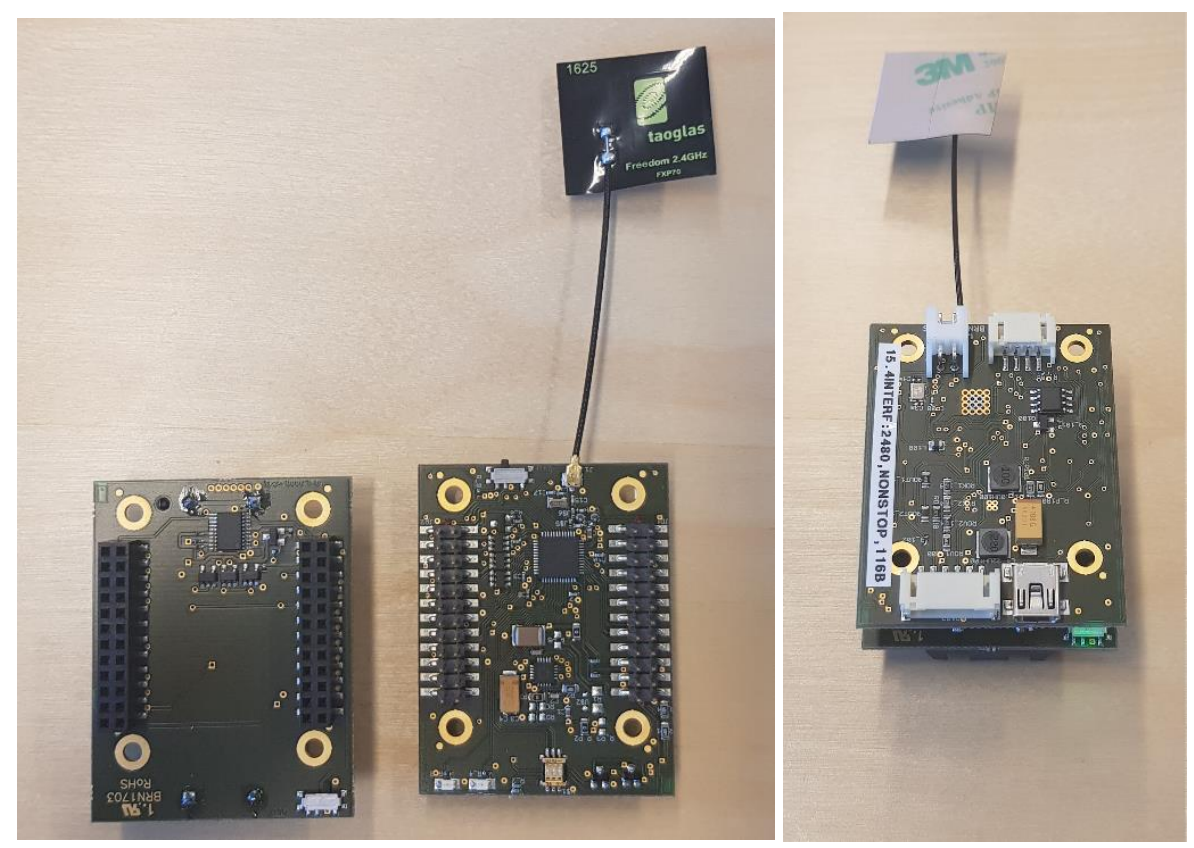

Figure 2. CWC-MOD-POW platforms 


\section{$5 \quad$ Results}

The developed analytical model was implement to Matlab and measurements were conducted to evaluate BLE PER under ZigBee interference. Table 1 shows the parameters used in analytical and experimental performance evaluation of BLE 4. In addition, measurement were done for BLE 5 coded $(S=8)$ mode with the same parameter settings to find out the gain provided by forward error correction.

Table 1. Parameters for analytical and experimental performance evaluation

\begin{tabular}{ll}
\hline Parameter & Value \\
\hline Number of interfering nodes & 3 \\
\hline Distance to interferers & Case $1=4 \mathrm{~m}$; Case2 $=6 \mathrm{~m}$ \\
\hline Desired BLE link length & $4-11 \mathrm{~m}$ \\
\hline Frequency & $2.480 \mathrm{GHz}(\mathrm{BLE}$ CH\#39, ZigBee CH\#26) \\
\hline BLE bandwidth, $B_{\mathrm{Ds}}$ & $1 \mathrm{MHz}$ \\
\hline ZigBee bandwidth, $B_{\mathrm{I}}$ & $2 \mathrm{MHz}$ \\
\hline Transmit power, BLE & $0 \mathrm{dBm}$ \\
\hline Transmit power, ZigBee & $0 \mathrm{dBm}$ \\
\hline Path loss exponent, $n$ & 1.2 \\
\hline RSSI at 1 m, BLE & $-15 \mathrm{dBm}$ \\
\hline ZigBee Tx to BLE Rx loss & $-9 \mathrm{dBm}$ \\
\hline Payload length, BLE & 12 octets \\
\hline Payload length, ZigBee & 116 octets \\
\hline Packet rate BLE node & One packet every $50 \mathrm{~ms}$ \\
\hline Packet rate ZigBee node & One packet every $5 \mathrm{~ms}$ \\
\hline Data Rate (BLE) & 1 Mbps \\
\hline Data Rate (ZigBee) & 250 kbps \\
\hline
\end{tabular}

Figure 3 shows PER results for the scenario where three ZigBee nodes are interfering LOS BLE link which length was varied. ZigBee nodes were set at 4 meters distance from BLE receiver to create LOS interference. From Figure 3 it can be observed that the effect of interference becomes visible in PER results when the BLE 4 link distance is longer than 5 meters. PER increases very rapidly when the BLE link distance is increased and reaches its maximum value when BLE link length is 10 meters. After that point, almost all packets are lost since interference is too strong in comparison to BLE 4 signal strength. As a reference result, in case without interference, the PER of BLE link remained below $15 \%$ for a link distance of 80 meters. Further it can be observed that the measurement results match well with the analytical results of BLE 4 . It must be noted that in analytical calculations we used $-9 \mathrm{dBm}$ loss for ZigBee to BLE receiver. The rationale for this negative gain is different antenna types and their orientations used in the ZigBee and BLE nodes, which are assumed to decrease the strength of experienced interference at BLE receiver. BLE 5 coded case measurement results of Figure 3 show that the error correction enables to maintain low PER until the link distance increases to longer than 9 meters, enabling 3 meters (50\%) higher communication range. After that point the PER increases rapidly also in the coded mode, i.e., the coding cannot correct the errors created by interference. 


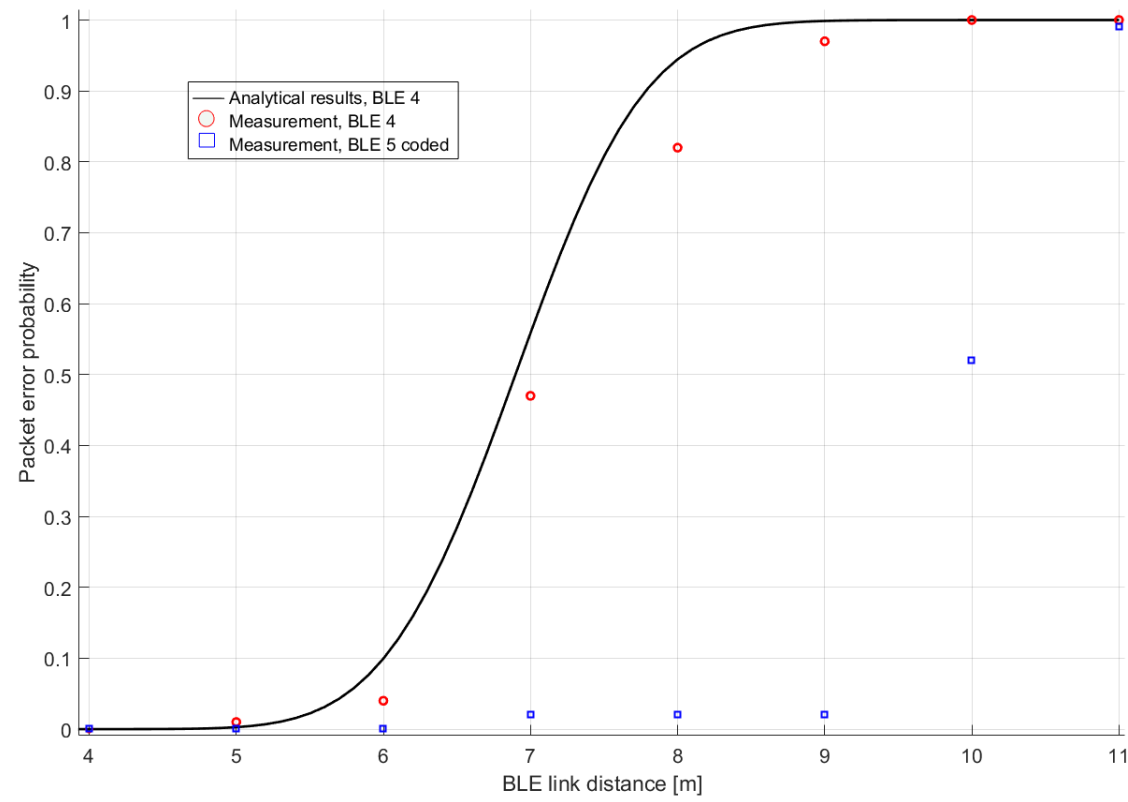

Figure 3. BLE PER under interference of three ZigBee nodes at $4 \mathrm{~m}$ distance.

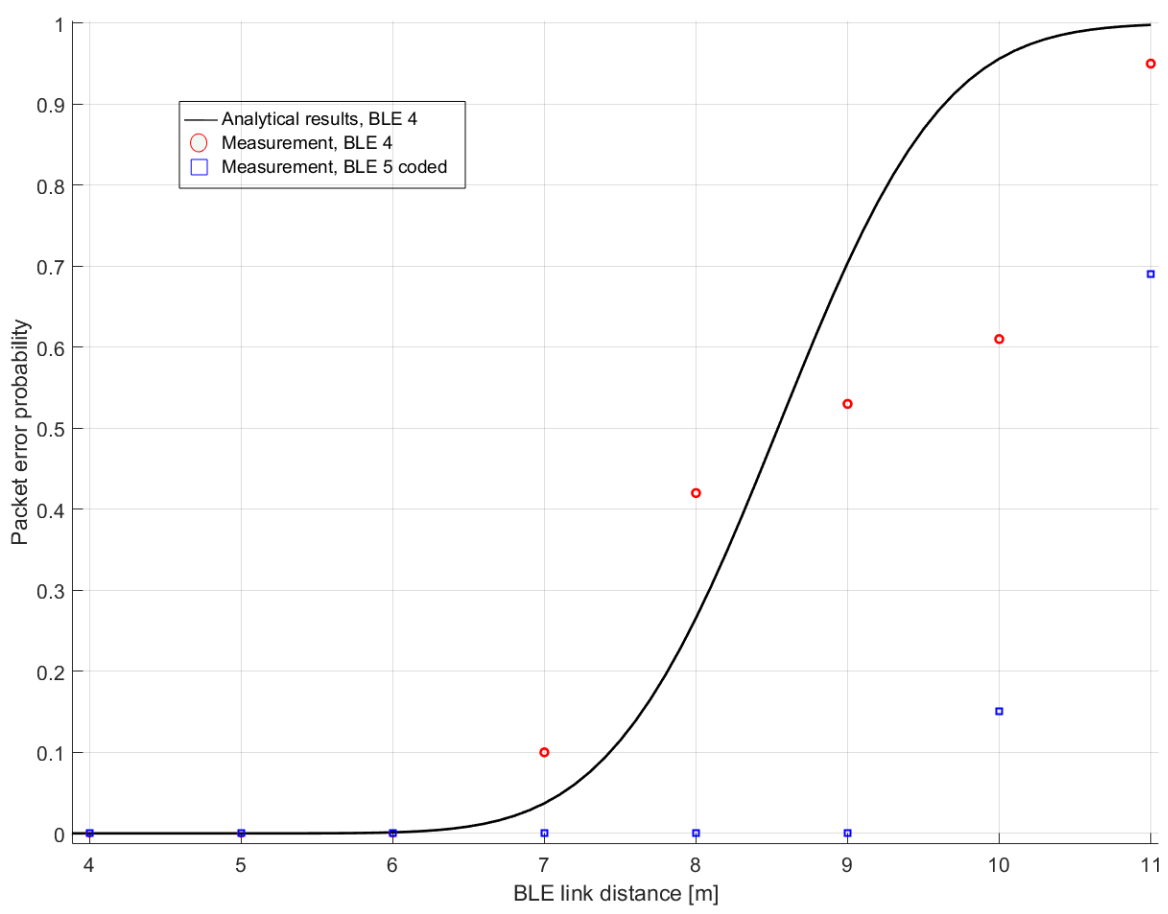

Figure 4. BLE PER under interference of three ZigBee nodes at $6 \mathrm{~m}$ distance. 
Figure 4 shows the PER for the case where ZigBee nodes were set at $6 \mathrm{~m}$ distance from BLE receiver. As expected, it can be observed that BLE 4 link can be longer in this case before the PER starts to increase due to interference. Also in this case it can be observed that the PER starts to increase rapidly when the BLE link length is increased beyond 6 meters. This result verifies that the analytical results are matching well with the measurement results even there is bit more variation in the results in comparison to Figure 3 case. BLE 5 coded mode measurement results show similar behavior to that in Figure 3, the coding gain being $(2-3 \mathrm{~m})$ in terms of increased BLE link length.

\section{Conclusions}

This paper reports the results of an experimental and analytical packet error rate evaluation of BLE under ZigBee interference. Analytical results were derived for the BLE 4 mode while measurement were conducted not only for BLE 4 but also for BLE 5 coded mode. Measurement results verified the analytical model results. Analytical model can be used to derive results also for other scenarios and as well as for other type of interferers.

Our results show that the worst-case interference is very harmful for BLE communication even when using the BLE 5 coded mode. Here the worst-case interference means that the interferers are at the same channel with the useful signal and full packet collisions occur. In terms of BLE link distance, the error correction coding gain was found to be only 2 to 3 meters, i.e., approximately one third of the used communication ranges. Results highlight that it is very important to pay attention to different technologies coexistence since the amount of IoT devices is increasing rapidly creating interference to each other.

Resilience towards interference is especially important in applications which require high reliability communications. Erroneous packet receptions will also decrease the energy efficiency which is highly important in IoT applications. Results of this paper show that the BLE communication performance will decrease drastically if there are interfering ZigBee nodes in a close vicinity $(<6 \mathrm{~m})$ at the same frequency channel. In future studies were are going to evaluate different coexistence scenarios using analytical modeling and experimental measurements.

\section{Acknowledgments}

This research has been financially supported in part by Academy of Finland 6Genesis Flagship (grant 318927). 


\section{References}

1. P. Lamkin, "Wearable Tech Market To Be Worth $\$ 34$ Billion By 2020," https://www.forbes.com/sites/paullamkin/2016/02/17/wearable-tech-market-to-be-worth-34billion-by-2020/\#4cbe76e83cb5 (2016)

2. Tractica, "Healthcare Wearable Device Shipments to Reach 98 Million Units Annually by 2021," https://www.tractica.com/newsroom/press-releases/healthcare-wearable-device-shipments-to-reach-98-million-units-annually-by-2021/ (2016)

3. R. Haydon, "Bluetooth Low Energy: The Developer's Handbook", Pearson Education Inc., New Jersey, USA, 2013.

4. IEEE Standard for Low-Rate Wireless Networks, "IEEE Std 802.15.4-2015 (Revision of IEEE Std 802.15.4-2011)" (2016)

5. ZigBee Alliance, http://www.zigbee.org/.

6. IEEE Std. 802.15.6: IEEE Standard for Local and metropolitan area networks-Part 15.6: Wireless Body Area Networks. Standard, The Institute of Electrical and Electronics Engineers, Inc. (2012)

7. M. Hämäläinen et al., "ETSI TC SmartBAN: Overview of the wireless body area network standard," International Symposium on Medical Information and Communication Technology (ISMICT) (2015)

8. H. Karvonen, M. Hämäläinen, J. Iinatti and C. Pomalaza-Ráez, "Coexistence of Wireless Technologies in Medical Scenarios," European Conference on Networks and Communications (EUCNC), Oulu, Finland (2017)

9. Nokia white paper, "LTE evolution for IoT connectivity," (2017)

10. H. Karvonen, C. Pomalaza-Ráez, K. Mikhaylov, M. Hämäläinen and J. Iinatti, "Interference of Wireless Technologies on BLE Based WBANs in Hospital Scenarios," IEEE International Symposium on Personal, Indoor and Mobile Radio Communications (PIMRC), Montreal, Canada (2017)

11. Bluetooth SIG, "Bluetooth Core Specification v 5.0" https://www.bluetooth.com/specifications/bluetooth-core-specification (2016)

12. Bluetooth SIG, "Bluetooth Core Specification 5.0 FAQ" https://www.bluetooth.com/ /media/files/specification/bluetooth-5-faq.ashx?la=en (2016)

13. R. de Francisco, "Indoor Channel Measurements and Models at $2.4 \mathrm{GHz}$ in a Hospital," IEEE Global Telecommunications Conference (GLOBECOM), Miami, FL, USA, 2010.

14. H. Karvonen, K. Mikhaylov, M. Hämäläinen, J. Iinatti and C. Pomalaza-Ráez, "Interference of wireless technologies on BLE based WBANs in hospital scenarios," International Symposium on Personal, Indoor, and Mobile Radio Communications (PIMRC), Montreal, QC, 2017.

15. R. Natarajan, P. Zand and M. Nabi, "Analysis of Coexistence between IEEE 802.15.4, BLE and IEEE 802.11 in the $2.4 \mathrm{GHz}$ ISM band," 42th Conference of the IEEE Industrial Electronics Society (IECON 2016), Florence, Italy, 2016, pp. 6025-6032.

16. Nordic nRF52840, https://www.nordicsemi.com/eng/Products/nRF52840.

17. K.Mikhaylov, "Plug and play reconfigurable solutions for heterogeneous IoT", Ph.D. thesis, University of Oulu, 2018, online http://jultika.oulu.fi/Record/isbn978-952-62-1841-0

18. K. Mikhaylov and J. Petäjäjärvi, "Design and Implementation of the Plug\&Play enabled Flexible Modular Wireless Sensor and Actuator Network Platform", Asian J. Control, Vol. 19, Issue 5, pp. 1-21, Sept. 2017.

19. Texas Instruments, CC2650, online http://www.ti.com/product/cc2650

20. Taoglas, FXP70 data sheet, online https://eu.mouser.com/datasheet/2/398/FXP70.07.0053A1219667.pdf 